\title{
Sigmoid growth curves, a new approach to study the dynamics of the epicotyl emergence of oak
}

\author{
Joanna Ukalska $\bowtie$, Szymon Jastrzębowski ${ }^{2}$ \\ ${ }^{1}$ Warsaw University of Life Sciences, Faculty of Applied Informatics and Mathematics, Department of Econometrics \\ and Statistics, Biometry Division, Nowoursynowska 159, 02-776 Warsaw, Poland, e-mail: joanna_ukalska@sggw.pl \\ ${ }^{2}$ Forest Research Institute, Department of Silviculture and Genetics of Forest Trees, Braci Leśnej 3, Sękocin Stary, \\ 05-090 Raszyn, Poland
}

\section{Abstract}

Three of the most frequently used sigmoidal growth curves from the Richards family are the logistic model, Gompertz model and Richards model. They are used in the analysis of organismal growth over time in many disciplines/studies and were proposed in many parameterisations. Choosing the right parameterisation is not easy. The correct parameterisation of the model should take into account such parameters that are useful to describe the analysed growth phenomenon and are biologically relevant without additional calculations. In addition, each parameter of the model only affects one shape characteristic of each growth curve, which makes it possible to determine standard errors and confidence intervals using statistical software.

Growth curves in germination dynamics studies should provide information on topics such as the length of the lag in onset of germination, the maximum germination rate and, when it occurs, the time at which $50 \%$ of seeds will germinate and the final germination proportion. In this article, we present three parameterisations of the logistic, Gompertz and Richards models and indicate two parameterisations for each model, corresponding to the abovementioned issues. Our proposition is parameterisation by taking into account the maximum absolute growth rate. Parameterisations indicated as useful for germination dynamics are characterised by the fact that each parameter has the same meaning in every model, so its estimates can be compared directly amongst the models. We also discussed the goodness-of-fit measures for nonlinear models and in particular measures of nonlinear behaviour of a model's individual parameters as well as overall measures of nonlinearity.

All described models were used to study the dynamics of the epicotyl emergence of pedunculate oak. After checking the close-to-linear behaviour of the studied model parameters and by taking into account the criteria of model selection $\left(\mathrm{AIC}_{\mathrm{c}}\right.$ of each growth curve and the residual variance $[\mathrm{RV}]$ ), the best model describing the dynamics of epicotyl appearance of pedunculate oak was the Richards curve.

\section{KeY WORDS}

cumulative germination, Gompertz model, growth curves, logistic model, nonlinear regression, pedunculate oak, Richards model 


\section{INTRODUCTION}

Growth curves are used to model the growth of an individual/organism or population (plants or animals) over time. Growth measurements (dependent variable) can be body mass, body length or mass/length of different parts of the body. Growth curves can have various growth patterns. If there is an upper limit, for example, because of environmental resources, growth proceeds sigmoidally and asymptotically. In the initial phase, growth is relatively slow but accelerates thereafter and decreases in the final phase, approaching the upper asymptote (Fig. 1). The processes taking place in trees can be described using this type of growth (Kramer and Kozlowski 1979).

There are numerous studies that used sigmoid (Sshaped) curves in growth analyses, and many growth models have been proposed. Amongst the most frequently used are logistic, Richards and Gompertz models. However, these models have been applied in various fields in many different notations and parameterisations
(Tjørve and Tjørve 2010, 2017a), making it difficult to choose the correct model for growth data analysis and to compare the results of a study with those already published. Tjørve and Tjørve (2010, 2017a, 2017b) reviewed a number of re-parameterisations of the Gompertz model and Richards model family and discussed their usefulness.

Some forms of growth curves are described by parameters that are not meaningful in the biological sense (Zwietering et al. 1990). To find the biological meaning of the parameter, an additional calculation is necessary. For example, for the logistic function given in the form

$$
Y(t)=\frac{A}{1+\exp (B-C t)}
$$

where $Y(t)$ and $t$ are variables and $A, B$ and $C$ are parameters, parameter $B$ has no direct biological meaning and can be used to calculate the time at inflection: $t_{i}=B / C$.

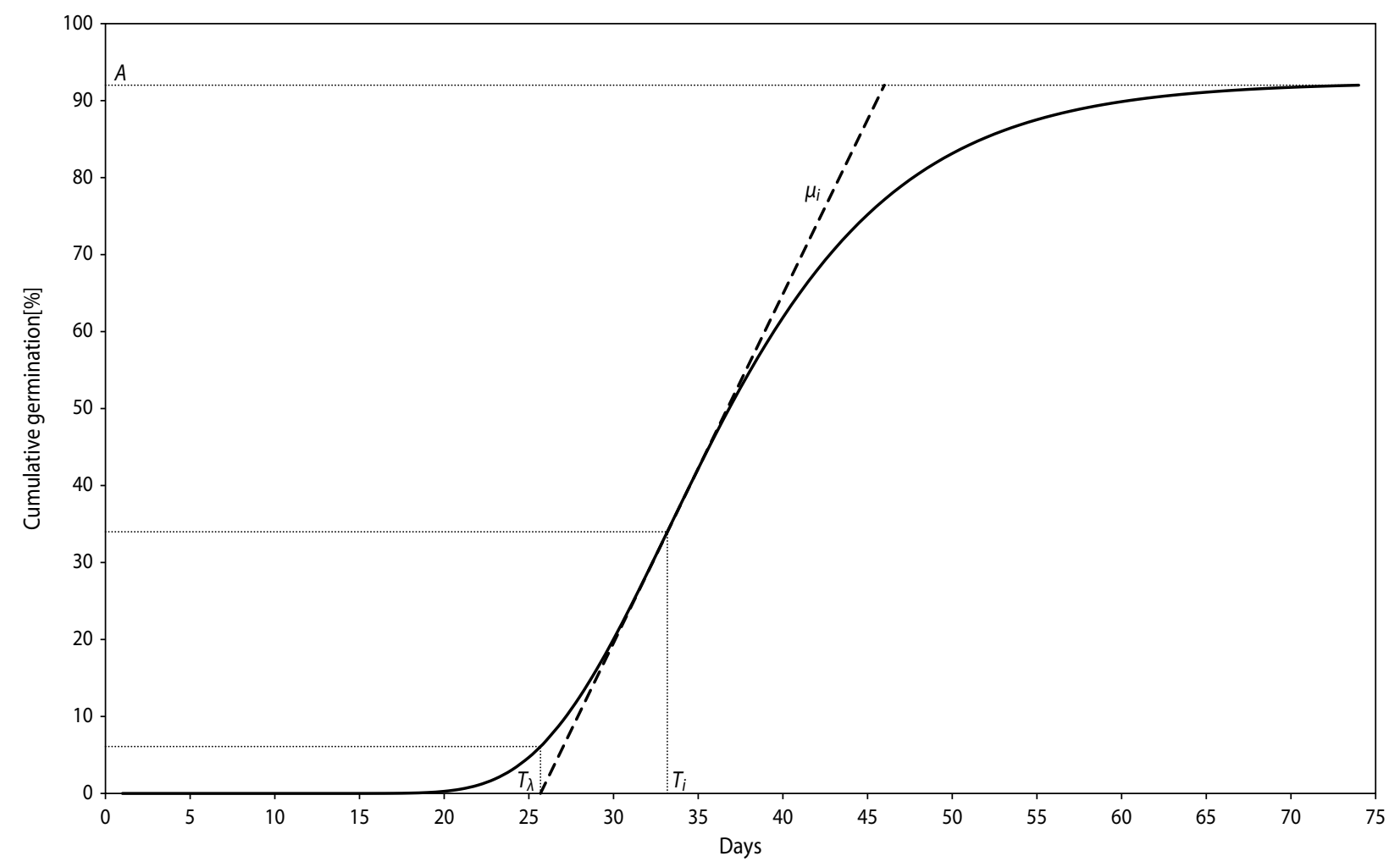

Figure 1. Shape characteristics of sigmoidal growth curve describing germination dynamics: $A$, upper asymptote; $\mu_{i}$, maximum absolute growth rate represented by the tangent at an inflection - slope at an inflection (dashed line); $T_{i}$, time at an inflection; $T_{\lambda}$, lag time 
The parameters of the growth function are estimated using various estimation methods and statistical software. However, it is difficult to calculate standard errors or the confidence intervals for the biological parameters if they are not estimated directly in the equation (Zwietering et al. 1990). Moreover, when determining parameters using statistical software, it is necessary to enter their initial values. It is difficult to estimate start values for the parameters if they have no biological meaning.

The basic feature describing the quality of seeds is the germination of seeds, that is, the percentage of seeds that germinate under the temperature and humidity conditions appropriate for a given species. Seed germination dynamics, that is, the germination process of woody plant seeds over time, is examined less frequently (Ashton and Larson 1995; Struve 1998; García-De La Cruz 2016; Hawkins 2018). The dynamics of germination as a function of the number of seeds germinated in a given unit of time can be described by a bell-shaped function. In this case, the cumulative germination capacity can be modelled using sigmoid growth curves. In the case of oak species belonging to the white oaks sections (Lepidobalanus), apart from germination, epicotyl emergency dynamics is also crucial. In the field, the appearance of the epicotyl (shoot) above the soil surface is the first sign that germination has occurred (Fenner and Thompson 2005). Analysis of germination dynamics (epicotyl emergence dynamic) provides answers to the following questions (the properties or parameters of the growth curves corresponding to the given stage of the germination process are given in brackets; Fig. 1):

1. How much time is needed to start the process of germination, that is, how large is the lag in the onset of germination (lag time)?

2. What is the maximum germination rate (slope at inflection)?

3. When does this occur (time at inflection)?

4. At what time will $50 \%$ of the seeds germinate $\left(T_{50}\right)$ ?

5. What is the final germination proportion, that is, germination capacity (upper asymptotic value)?

In this context, the purpose of this study is to present growth curves from the Richards family, with particular regard to those parameterisations/forms that are useful for modelling germination dynamics. We will present such growth model forms whose parameters can be comparable across models. Amongst the dis- cussed models, three models are proposed by us. The proposed models will be applied to study the dynamics of the epicotyl emergence of pedunculate oak (Quercus robur L.).

\section{MAterial AND MEthodS}

\section{Sigmoid growth curves}

\section{Logistic function}

A logistic curve describing the population growth was proposed and named by P. F. Verhulst (Verhulst 1838, 1845) to describe the growth in the size of a population or organ. One of the most frequently used forms of the logistic model is ( $k$ form; Model 1$)$ :

$$
C G(t)=\frac{A}{1+\exp \left[-k\left(t-T_{i}\right)\right]}
$$

where:

$C G(t)$ - the expected cumulative percentage germination at time $t$,

$A \quad$ - the upper asymptote (theoretical maximum for $C G(t))$,

$k \quad-$ the growth-rate coefficient, shape parameter,

$T_{i} \quad$ - the time at an inflection.

The position of the inflection point, that is, the time at which germination reaches the maximum rate of $T_{i}$, is given directly in the model. Parameter $T_{i}$ is a location parameter that shifts the growth curve horizontally without changing it shape. Logistic function is very similar to the cumulative normal distribution, that is, unskewed and perfectly symmetrical. It is symmetrical about the point of inflection, whose coordinates are $\left(T_{i}, A / 2\right)$. This means that $T_{i}$ indicates the germination time of $50 \%$ of seeds $\left(T_{50}\right)$ from those that germinate (Tab. 1). For growth models, the growth-rate coefficient $k$ is always positive, and larger values indicate a quicker rise from zero to the upper asymptote $A$. In many studies of organismal growth, parameter $k$ is used as the relative growth rate at inflection, thus the maximum relative growth rate (i.e. at inflection and relative to maximum $G C$ value). Tjørve and Tjørve (2017a) indicated that this is an incorrect interpretation of parameter $k$. To find the maximum relative growth rate, parameter $k$ should be divided by 
$4(k / 4)$ and the maximum absolute growth rate can be calculated as $\frac{A k}{4}$.

To avoid recalculation of the $k$ parameter to obtain the biological meaning, we propose re-parameterisation of the logistic model in the following form $\left(\mu_{i}\right.$ form; Model 2):

$$
C G(t)=\frac{A}{1+\exp \left[-\frac{4 \mu_{i}}{A}\left(t-T_{i}\right)\right]}
$$

In Model 2, instead of $k$, it is $\frac{4 \mu_{i}}{A}$. The parameter $\mu_{i}$ represents the maximum absolute growth rate, that is, the slope of the tangent at the inflection point, whilst the meaning of the other parameters is analogous to that of Model 1. An important advantage of this reparameterisation is that the upper asymptote $A$ does not affect parameter $\mu_{i}$. This means that this parameter shows the maximum percentage of germinated seeds per unit of time without reference to the final percentage of germinated seeds, which is given by $A$. This makes it possible to compare the germination rates of different data sets.

The third parameterisation of the logistic model, which may be useful for studying germination dynamics, is the model proposed by Zwietering et al. (1990) in the following form ( $T_{\lambda}$ form; Model 3):

$$
C G(t)=\frac{A}{1+\exp \left[-\frac{4 \mu_{i}}{A}\left(t-T_{\lambda}\right)+2\right]}
$$

in which, instead of time at inflection $T_{i}$, there is parameter $T_{\lambda}$, termed 'lag time' and describing the transition from the low period of germination (lag period), after which the germination rate considerably increases. The lag time $T_{\lambda}$ occurs for $t=T_{\lambda}$ when

$$
C G(t)=\frac{A}{1+e^{2}}
$$

that is, always falls at $11.92 \%$ of the upper asymptote $A$.

\section{Gompertz function}

Actuary B. Gompertz, presenting his law of human mortality (Gompertz, 1825), proposed using the exponential function to describe the relationship between increasing death rate and age. Gompertz presented only the prob- ability density function, whilst Makeham (1873) stated the well-known cumulative form of this model. In the literature, numerous forms of the Gompertz model can be found, and a detailed review of the Gompertz model was presented by Tjørve and Tjørve (2017a). One of the most used forms of the model is ( $k$ form; Model 4$)$ :

$$
C G(t)=A \cdot \exp \left\{-\exp \left[-k\left(t-T_{i}\right)\right]\right\}
$$

The meaning of the parameters is the same as for Models 1 and 2. The shape parameters $A$ and $k$ change the curve shape but leave the value of the location parameter $T_{i}$ unaltered. The parameter $T_{i}$ controls the time at which the inflection occurs. The Gompertz model is not symmetrical about the point of inflection, whose coordinates are $\left(T_{i}, A / e\right)$, which means that by the time $T_{i}$, $36.8 \%$ of the germinating seeds will germinate, whilst $A / 2$ seeds will germinate until time $t=T_{i}-\log (\log (2)) / k=$ $=T_{i}+0.3665 / k$ (Tab. 1). Maximum relative growth rate and maximum absolute growth rate $\mu_{i}$ can be calculated from Model 4 by dividing $k$ by the base of the natural logarithm $k / e$ and $k A / e$, respectively. To obtain $\mu_{i}$ directly from the Gompertz model, we propose re-parameterisation, which is given as ( $\mu_{i}$ form; Model 5)

$$
C G(t)=A \cdot \exp \left\{-\exp \left[-\frac{e \mu_{i}}{A}\left(t-T_{i}\right)\right]\right\}
$$

in which parameter $k$ is replaced by $\frac{e \mu_{i}}{A}$. As in Equation 2, each parameter of Model 5 controls one curve characteristic. The third form of the model, including the $T_{\lambda}$ parameter (Zwietering et al. 1990), can be given as $\left(T_{\lambda}\right.$ form; Model 6)

$$
C G(t)=A \cdot \exp \left\{-\exp \left[-\frac{e \mu_{i}}{A}\left(t-T_{\lambda}\right)+1\right]\right\}
$$

Here, $T_{\lambda}$ falls when $C G(t)=A \cdot \exp (-e)$, that is, a lag time always occurs at the $6.6 \%$ of the upper asymptote $A$ (Tjørve and Tjørve 2017a).

\section{Richards function}

The Richards model (Richards 1959), also called as generalised logistic function, allows for flexibility in the asymmetry by including an additional parameter, $d$, which controls which asymptote is nearest to the inflection point. The form of the model that is useful for studying the dynamics of germination is as follows 
(parameters are the same as for Models 1, 2, 4 and 5) ( $k$ form; Model 7):

$$
C G(t)=\frac{A}{\left\{1+d \cdot \exp \left[-k\left(t-T_{i}\right)\right]\right\}^{1 / d}}
$$

When testing germination dynamics according to Model 7, parameter $d$ will have positive values. The inflection point is floating and can be given as a proportion of the upper asymptote $A$. This proportion is determined by the parameter $d$ and falls at $A /(1+d)^{1 / d}$ (Tjørve and Tjørve 2010). When $d<1$, less than half of the seeds will germinate before time $T_{i}$ (e.g. as in the Gompertz curve); when $d=1$, half of the seeds will germinate before $T_{i}$ and half afterwards (i.e. as in the logistic curve); when $d>1$, more than half of the seeds will germinate before $T_{i}$. Also, $A / 2$ seeds $\left(T_{50}\right)$ will germinate until time

$$
t=\frac{k T_{i}-\log \left[\left(2^{d}-1\right) / d\right]}{k}
$$

(Tab. 1).

The Richards model, taking into account the maximum absolute growth rate $\mu_{i}$, derived from Model 7, has the following form ( $\mu_{i}$ form; Model 8):

$$
C G(t)=\frac{A}{\left\{1+d \cdot \exp \left[-\frac{\mu_{i}}{A}(1+d)^{(1+1 / d)}\left(t-T_{i}\right)\right]\right\}^{1 / d}}
$$

Parameter $k$ in Model 7 is replaced by $\frac{\mu_{i}}{A}(1+d)^{(1+1 / d)}$. The Richards model, taking into account the parameters $\mu_{i}$ and $T_{\lambda}$ (Zwietering et al. 1990), is given as $\left(T_{\lambda}\right.$ form; Model 9):

$$
C G(t)=\frac{A}{\left\{\begin{array}{l}
1+d \cdot \exp (1+d) . \\
\exp \left[-\frac{\mu_{i}}{A}(1+d)^{(1+1 / d)}\left(t-T_{\lambda}\right)\right]
\end{array}\right\}^{1 / d}}
$$

All growth models presented above (1-9) represent the Richards model family (Tjørve and Tjørve 2010, 2017a, 2018). Models 7-9 have the most general form amongst the models presented in these studies. Models 1-6 can be derived from Models 7-9. With $d=1$, Models 7-9 become the logistic Models 1-3, respectively. The Gompertz model is also a special case of the Richards model (Seber and Wild 1989). However, the Richards model is not reduced to the Gompertz model by simply substituting a given value for $d$ (Tjørve and Tjørve 2010). When $d \rightarrow 0$ but $d \neq 0$, Models 7-9 converge towards the Gompertz Models 4-6.

The advantage of re-parameterisations presented as Models 2-3, 5-6 and 8-9 is that their parameter estimates are comparable between these models. The maximum absolute growth rate $\mu_{i}$ is comparable amongst all mentioned models; $T_{i}$ is comparable amongst Models 2,

Table 1. Formulas allowing to determine $T_{50}(A / 2)$ based on the considered parameterisations of growth curves; $\mathrm{m} 1-\mathrm{m} 9 \mathrm{refer}$ to Models 1-9; log, the natural logarithm

\begin{tabular}{|l|c|c|c|c|c|c|}
\cline { 2 - 6 } \multicolumn{1}{l|}{} & \multicolumn{2}{|c|}{ Logistic } & \multicolumn{2}{c|}{ Gompertz } & $\mathrm{m} 7$ & $\frac{k T_{i}-\log \left(\frac{2^{d}-1}{d}\right)}{k}$ \\
\hline$k$ form & $\mathrm{m} 1$ & $T_{i}$ & $\mathrm{~m} 4$ & $T_{i}-\frac{\log (\log 2)}{k}=T_{i}+0.3665 k$ & & $\frac{\mu_{i}}{A}(1+d)^{(1+1 / d)} T_{i}-\log \left(\frac{2^{d}-1}{d}\right)$ \\
\hline$\mu_{i}$ form & $\mathrm{m} 2$ & $T_{i}$ & $\mathrm{~m} 5$ & $T_{i}-\frac{A \log (\log 2)}{e \mu_{i}}=T_{i}+0.1348 \frac{A}{\mu_{i}}$ & $\mathrm{~m} 8$ & $\frac{\mu_{i}}{A}(1+d)^{(1+1 / d)}$ \\
$T_{\lambda}$ form & $\mathrm{m} 3$ & $\frac{A}{2 \mu_{i}}+T_{\lambda}$ & $\mathrm{m} 6$ & $T_{\lambda}+\frac{A[1-\log (\log 2)]}{e \mu_{i}}=T_{\lambda}+0.5027 \frac{A}{\mu_{i}}$ & $\mathrm{~m} 9$ & $\frac{1+d+\frac{\mu_{i}}{A}(1+d)^{(1+1 / d)} T_{\lambda}-\log \left(\frac{2^{d}-1}{d}\right)}{\frac{\mu_{i}}{A}(1+d)^{(1+1 / d)}}$ \\
\hline
\end{tabular}


5 and 8 ; and $T_{\lambda}$ is comparable amongst Models 3, 6 and 9. In addition, achieving the maximum relative growth rate is easy from any of Models $2-3,5-6$ and 8-9. The $\mu_{i}$ value should be divided by the value of the upper asymptote $A$. The value $A / 2=T_{50}$ can be determined based on each of the 1-9 models, using the formulas in Table 1 . The $k$ parameter is not directly comparable amongst the most frequently used traditional models, that is, Models 1, 4 and 6.

\section{Estimation of the model parameters}

The estimation of growth curves is performed using the nonlinear least squares method according to one of the algorithms: Gauss-Newton, Newton, steepest descent (gradient) and Levenberg-Marquardt (Marquardt 1963; Dennis and Schnabel 1983). The nonlinear least squares method is an iterative method, and therefore the appropriate initial values of the parameters of the model must be chosen so that the algorithm converges. There is no standard procedure for obtaining initial estimates. The most obvious and easiest method is to find the crude initial values based on a scatterplot of the growth data. The upper asymptote $A$ can be estimated this way. The coordinates of the point of inflection can then provide starting values for $T_{i}$. The value of parameter $\mu_{i}$ can be found as the largest difference between the percentage of germinated seeds between two consecutive measurements, that is, days. When testing germination dynamics, the parameter $d>0$ and its value depend on the point of inflection. The initial value of this parameter can be determined by checking if half of the seeds $(A / 2)$ germinated before time $T_{i}$ (then $d \in(0 ; 1)$, in time $T_{i}$ (then $d=1$ ) or after time $T_{i}$ (then $d>1$ ). In turn, the initial value of $T_{\lambda}$ can be taken for time $t$ when the percentage of germinated seeds is between $6 \%$ and $12 \%$ of the upper asymptote $A$, depending on the growth function one wants to use.

\section{Goodness-of-fit measures of nonlinear behaviour}

The least squares parameter's estimators in linear regression models are unbiased, are normally distributed and achieve the minimum possible variance. Such properties are generally agreed on to be the most desirable properties that an estimator can possess (Seber and Wild 1989). In nonlinear regression models, the least squares estimators may not have the aforementioned properties of a good estimator, especially with very small sample sizes. Nonlinear behaviour of model parameters is undesirable because the parameter estimates and their standards errors will be biased, leading to incorrect inferences (Ratkowsky 1983, 1990; Ratkowsky and Reddy 2017). Ratkowsky (1983) proposed the term 'close-to-linear' for model estimators that achieve the desirable properties.

Amongst nonlinearity measures, one can distinguish nonlinear measures used for testing of nonlinear behaviour, focusing on the model's individual parameters as well as global measures of nonlinearity. The first group includes Box's bias (b) (Box 1971) and Hougaard's skewness $(h)$ (Hougaard 1982, 1985) of the least squares parameter estimates, and the second group includes curvature measures of nonlinearity (Bates and Watts 1980). According to Ratkowsky, the value of the Box's bias for the close-to-linear parameter estimator should not exceed 1\% (Ratkowsky 1983). In turn, the measure of skewness indicates a close-to-linear behaviour of the parameter if its value is less than 0.25 , whereas, if the measure is greater than 1, a considerable nonlinear behaviour is present (Ratkowsky 1990). Haines et al. (2004) suggested that the model is reasonably close-to-linear if the absolute value of $h$ does not exceed 0.15. In turn, Ratkowsky (2017) proposed a compromise cut-off criterion, where the absolute value of the skewness measure $|h| \leq 0.2$ indicates 'good' behaviour, $0.2<|h| \leq 0.5$ indicates 'moderate' nonlinearity and $|h|>0.5$ indicates 'bad', that is, far-from-linear behaviour.

The second type of measures, overall measures of nonlinearity, consists of two components: the intrinsic and the parameter-effects curvatures (Bates and Watts 1980). The intrinsic curvature is measured using the root-mean-square intrinsic (RMS IN) and cannot be affected by re-parameterisation of the model. However, the parameter-effects curvature expressed as root-mean-square parameter-effects (RMS PE) can be affected by altering the parameterisation of the model. Ratkowsky examined that RMS IN is typically smaller than RMS PE (Ratkowsky 1983). These parameters are assessed using a critical curvature value (Bates and Watts 1980), which is a function of the sample size, the number of parameters of the model and a significance level $\alpha$. 


\section{Model selection}

When several growth models are fitted to the same data set, model selection is carried out based on the model-fit criteria. One of the more frequently used ones is the Akaike information criterion (AIC; Akaike 1974), which can be used when all candidate models have the same number of parameters and the sample size is large enough. Burnham and Anderson (2002) suggested using the AIC when the ratio $n / K$ ( $n$ is the sample size, $K$ is the number of parameters) is about 40 . If the models are compared with different numbers of parameters, the $\mathrm{AIC}_{\mathrm{c}}$ criterion takes into account the number of parameters, and penalising models with more parameters should be used.

\section{Epicotyl emergence experiment}

For the epicotyl emergence experiment, acorns from a selected seed stand of pedunculate oak ( $Q$. robur L.) were collected. The stand is located in the Strzelce Forest District (southeastern Poland), IV Nature-Forestry Country. In 2016, from underneath 10 sample trees, we harvested circa $5 \mathrm{~kg}$ of acorns. For the experiment, 60 acorns were randomly chosen and sown into the plastic boxes filled with sand. Before sowing, the acorns were stored at $3^{\circ} \mathrm{C}$ for 14 days (cold stratification to epicotyl dormancy break). The pots were placed in a germination room with constant air temperature $\left(21^{\circ} \mathrm{C}\right)$. After 2 weeks, only acorns with a visible radicle $(<2 \mathrm{~cm})$ were selected and transferred into pots containing a mixture of peat and sand (ratio 1:2); the radicle was not damaged. Pots were placed in growing chambers with simulated growth conditions for April, May and June (average and forecasted values considering predicted changes in the climate). Epicotyl emergence was recorded every day for a period of 74 days.

The data obtained in the experiment and used to analyse the epicotyl emergence dynamics were presented as an observed cumulative fraction epicotyls $C G(t)$, which have emerged until day $t$. Growth curves (Models 1-9) and measures of nonlinear behaviour of model parameters were obtained using the NLIN procedure of SAS/STAT ${ }^{\circledR} 14.3$ (SAS Institute Inc. 2017).

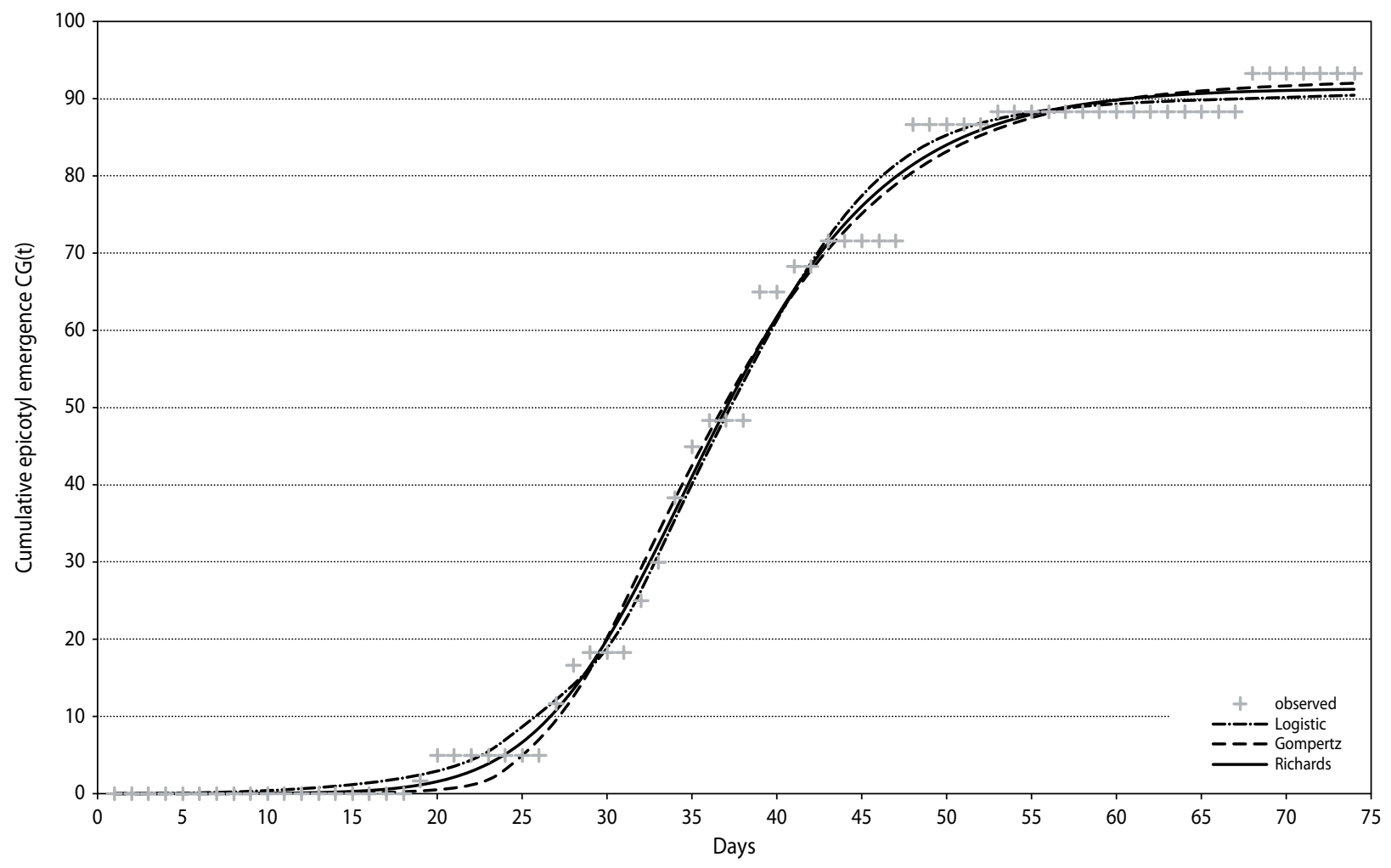

Figure 2. Fitted growth curves from the Richards family and experimental data describing the dynamics of the epicotyl emergence of pedunculate oak 


\section{Results}

Growth curves from the Richards family were determined for the studied data set of pedunculate oak (Fig. 2; Tab. 2 and 3). All tested models were significant $(\mathrm{p}<0.001)$. The best-fitting model was the Richards model, as its $\mathrm{AIC}_{\mathrm{c}}$ value, as well as RV, was lower than that for the other two models (Tab. 2). The values of RMS IN and RMS PE allow the investigation of an overall nonlinearity measure of the full set of parameters in each model. All models had nonsignificant RMS IN and RMS PE values, meaning that all tested models showed in general close-to-linear behaviour in model/ data set combination (Ratkowsky 1983, 2017).

To assess the estimation efficiency of each parameter of the model separately, Hougaard's measure of skewness $h$ and Box's measure of bias $b$ were determined (Tab. 3). For almost all parameters of the tested models, $|h| \leq 0.2$, indicating linear behaviour of these parameters. Only the parameter $k$ in Model 7 had $|h| \geq$ 0.2 , indicating moderate nonlinearity. Even considering the more conservative cut-off proposed by Haines et al. (2004) of $|h| \leq 0.15$ for linear behaviour, only one parameter exceeded this cut-off ( $d$ in the Richards model). Linear behaviour of the parameters of the tested data set was also found with Box's bias $b$, whose values for all parameters, except one, were less than $1 \%$. Only for parameter $d$ in the Richards model, $b$ slightly exceeded 1\% (Tab. 3).

The parameters estimated for Models 1-9, given in Table 3, are comparable amongst the models. The highest value of the upper asymptote $A$ was obtained for the
Gompertz model and the smallest for the logistic model. However, based on the confidence intervals for parameter $A$, it can be observed that the differences between $A$ values were not significant. The values of maximum absolute growth rate $\mu_{i}$ and lag time $T_{\lambda}$ were similar for all models. When comparing $T_{i}$ values, it should be remembered that they show time at inflection, whose location results from the properties of a given model. For the same data, values for the Gompertz model (inflection point for $C G\left(T_{i}\right)=A / e=0.368 A$ ) will always be smaller than those for the logistic model (inflection point for $C G\left(T_{i}\right)=A / 2$ ). For the Richards model, the inflection point was between the Gompertz and logistic models because for the analysed data set, $A /(1+d)^{1 / d}=$ $=0.433 \mathrm{~A}$. On the basis of the fitted growth functions, the time until $50 \%$ of seeds germinate $\left(T_{50}\right)$ was determined (Tab. 3). The $T_{50}$ values are very similar, and the difference between them did not exceed 0.3 days. The largest value of the parameter $k$ was obtained for the logistic model and the smallest for the Gompertz model. The confidence interval for this parameter in the logistic model did not overlap with the confidence intervals determined for other models. It should be noted, however, that the parameter $k$ is not readily interpretable because it does not directly answer any of the characteristics of the growth curve, which is directly relevant to the germination dynamics analysis, that is, answering questions $1-5$ referred to in the Introduction section. To obtain the maximum absolute growth rate, additional calculations have to be carried out, which are different for each of the tested models (see Subsection 1 in the Methods section).

Table 2. Goodness of fit of the models: $\mathrm{AIC}_{\mathrm{c}}$ and residual variance $(\mathrm{RV})$ and assessment of nonlinear behaviour: intrinsic (RMS IN) and parameter-effects (RMS PE) curvatures of the models as well as curvature critical value $\mathrm{P}_{c} ; \mathrm{m} 1-\mathrm{m} 9 \mathrm{refer}$ to Models 1-9

\begin{tabular}{|c|c|c|c|c|c|c|c|c|c|}
\hline Parameter & $\mathrm{m} 1$ & $\mathrm{~m} 2$ & $\mathrm{~m} 3$ & $\mathrm{~m} 4$ & $\mathrm{~m} 5$ & $\mathrm{~m} 6$ & $\mathrm{~m} 7$ & $\mathrm{~m} 8$ & $\mathrm{~m} 9$ \\
\hline $\mathrm{AIC}_{\mathrm{c}}{ }^{\mathrm{a}}$ & \multicolumn{3}{|c|}{-206.17} & \multicolumn{3}{|c|}{-209.37} & \multicolumn{3}{|c|}{-214.00} \\
\hline $\mathrm{RV}^{\mathrm{ab}}$ & \multicolumn{3}{|c|}{0.0797} & \multicolumn{3}{|c|}{0.0763} & \multicolumn{3}{|c|}{0.0706} \\
\hline $\mathrm{RMS} \mathrm{IN}^{\mathrm{a}}$ & \multicolumn{3}{|c|}{$0.0328^{\mathrm{ns}}$} & \multicolumn{3}{|c|}{$0.0378^{\mathrm{ns}}$} & \multicolumn{3}{|c|}{$0.0814^{\mathrm{ns}}$} \\
\hline RMS PE & $0.0646^{\text {ns }}$ & $0.0592^{\text {ns }}$ & $0.1100^{\mathrm{ns}}$ & $0.0832^{\mathrm{ns}}$ & $0.0727^{\text {ns }}$ & $0.1174^{\mathrm{ns}}$ & $0.3754^{\mathrm{ns}}$ & $0.3642^{\mathrm{ns}}$ & $0.1945^{\mathrm{ns}}$ \\
\hline$P_{c}$ & \multicolumn{6}{|c|}{$0.6048^{c}$} & \multicolumn{3}{|c|}{$0.6321^{\mathrm{d}}$} \\
\hline
\end{tabular}

${ }^{\text {a }}$ Models $1-3,4-6$ and 7-9 gave the same results for $\mathrm{AIC}_{\mathrm{c}}$, RV and RMS IN.

${ }^{\mathrm{b}} \mathrm{RV}$ values were multiplied by $10^{2}$.

${ }^{\text {ns }}$ Not significant.

${ }^{\mathrm{c}}$ Critical value for models with three parameters (Models 1-6).

${ }^{\mathrm{d}}$ Critical value for models with four parameters (Models 7-9). 
Table 3. Parameter estimates, their standard errors (SE), confidence intervals and parameter measures of nonlinearity $(h$, absolute values of the Hougaard's skewness; $b$, Box's bias [\%]) as well as $T_{50}$ values calculated from model parameters, m1-m9 refer to Models 1-9

\begin{tabular}{|c|c|c|c|c|c|c|c|}
\hline Model & Parameter & Estimate & Approximated SE & \multicolumn{2}{|c|}{$\begin{array}{l}\text { Approximate } 95 \% \\
\text { Confidence Limits }\end{array}$} & $\mathrm{h}$ & $\mathrm{b}$ \\
\hline \multirow{3}{*}{$\mathrm{m} 1$} & $A$ & 0.903 & 0.006 & 0.891 & 0.916 & 0.028 & 0.008 \\
\hline & $k$ & 0.203 & 0.007 & 0.189 & 0.217 & 0.144 & 0.110 \\
\hline & $T_{i}$ & 36.219 & 0.198 & 35.824 & 36.614 & 0.012 & 0.003 \\
\hline $\mathrm{m} 2^{\mathrm{a}}$ & $\mu_{i}$ & 0.046 & 0.001 & 0.043 & 0.049 & 0.145 & 0.110 \\
\hline \multirow{2}{*}{$\mathrm{m} 3^{\mathrm{a}}$} & $T_{\lambda}$ & 26.378 & 0.348 & 25.683 & 27.073 & 0.041 & 0.003 \\
\hline & $T_{50}$ & 36.219 & & & & & \\
\hline \multirow{3}{*}{$\mathrm{m} 4$} & $A$ & 0.924 & 0.007 & 0.909 & 0.939 & 0.061 & 0.012 \\
\hline & $k$ & 0.134 & 0.005 & 0.124 & 0.143 & 0.129 & 0.110 \\
\hline & $T_{i}$ & 33.176 & 0.187 & 32.804 & 33.549 & 0.005 & 0.006 \\
\hline $\mathrm{m}^{\mathrm{b}}$ & $\mu_{i}$ & 0.045 & 0.001 & 0.043 & 0.048 & 0.135 & 0.100 \\
\hline \multirow{2}{*}{$\mathrm{m} 6^{\mathrm{b}}$} & $T_{\lambda}$ & 25.690 & 0.324 & 25.044 & 26.336 & 0.044 & 0.001 \\
\hline & $T_{50}$ & 35.920 & & & & & \\
\hline \multirow{4}{*}{$\mathrm{m} 7$} & $A$ & 0.914 & 0.007 & 0.899 & 0.929 & 0.116 & 0.030 \\
\hline & $k$ & 0.160 & 0.013 & 0.135 & 0.186 & 0.280 & 0.370 \\
\hline & $d$ & 0.410 & 0.170 & 0.071 & 0.749 & 0.189 & 1.080 \\
\hline & $T_{i}$ & 34.665 & 0.575 & 33.518 & 35.811 & 0.121 & 0.040 \\
\hline $\mathrm{m} 8^{\mathrm{c}}$ & $\mu_{i}$ & 0.045 & 0.001 & 0.042 & 0.048 & 0.131 & 0.230 \\
\hline \multirow{2}{*}{$\mathrm{m} 9^{\mathrm{c}}$} & $T_{\lambda}$ & 25.870 & 0.349 & 25.175 & 26.566 & 0.004 & 0.045 \\
\hline & $T_{50}$ & 36.044 & & & & & \\
\hline
\end{tabular}

${ }^{\text {a }}$ Other parameters in Models 2 and 3 are the same as in Model 1.

${ }^{\mathrm{b}}$ Other parameters in Models 5 and 6 are the same as in Model 4.

${ }^{c}$ Other parameters in Models 8 and 9 are the same as in Model 7.

After checking the close-to-linear behaviour of the parameters of the growth models studied (Tab. 2 and 3) and taking into account the $\mathrm{AIC}_{\mathrm{c}}$ as well as the RV values (Tab. 2), the best model describing the dynamics of the epicotyl appearance of pedunculate oak is the Richards curve (Fig. 2). To describe the dynamics and answer questions 1-5 asked in Introduction section, Models 8 and 9 were most suitable. The time until intensive epicotyl emergence (lag time, $T_{\lambda}$ ) was 25.9 days. The maximum absolute growth rate of epicotyl emergence $\left(\mu_{i}\right)$ was about $4.5 \%$ and was achieved in $T_{i}=34.7$ days. The time until $50 \%$ of epicotyls appeared $\left(T_{50}\right)$ was 36 days. Total epicotyl emergence capacity was $91.4 \%$. Approximate standard errors and confidence intervals for the parameters described above are given in Table 3 .

\section{Discussion}

All the presented growth models (Models 1-9) belong to the Richards model family. As indicated in the Methods section, Models 7-9 can be derived from Models 1-3 as well as from Models 4-6. In addition, the models proposed by us, that is, Models 2, 5 and 8, are equivalent to models from the unified Richards family proposed by Tjørve and Tjørve (2010, 2017b), that is, Model 2 is equivalent to the $T_{i}$ form of the U-logistic model, Model 5 to the $T_{i}$ form of the U-Gompertz model and Model 8 to the $T_{i}$ form of the U-Richards model. Using the aforementioned models for a given data set, we obtained the same upper asymptote $A$ and time at inflection $T_{i}$. The difference is that, instead of the maximum absolute growth rate $\mu_{i}$, which occurs in our models, in $T_{i}$ forms of the U-models, the maximum relative growth 
rate is $k_{U}$. To obtain $T_{i}$ forms, proposed by Tjørve and Tjørve (2017b), from our model forms, it is sufficient to divide $\mu_{i}$ by the upper asymptote $A$. Between the $T_{i}$ form of the U-Richards model and the Richards model proposed by us (Model 8), there is also a difference regarding parameter $d$, which determines when the inflection occurs (Vrana et al. 2018). In our model, $d>0$, whilst in the $T_{i}$ form of the U-Richards model, $d>1$. The parameter $d$, however, has no interpretative meaning.

The sigmoid growth models proposed here (2-3; $5-6$; 8-9) allow an easy interpretation of all model parameters, because each parameter affects a single characteristic of each growth curve. This is a great advantage of these models (Tjørve and Tjørve 2010, 2017a, 2017b; Vrana 2018). The parameters $\mu_{i}$ (affects slope) and $A$ (represents upper asymptote) are shape characteristics, whilst $T_{i}$ (represents time at inflection) and $T_{\lambda}$ (lag time) are location parameters. In addition, as we noted above, the maximum relative growth rate can easily be calculated based on $\mu_{i}$. In Methods section, we also specified how to determine $T_{50}$ (time to germination of $50 \%$ of seeds) based on each of Models 1-9 (Tab. 1).

The selection of one of the two parameterisations of the growth curves, taking into account the different location parameters, that is, parameter $T_{i}$ (Models 2, 5 and 8) or parameter $T_{\lambda}$ (Models 3, 6 and 9), depends on which parameter value is most convenient to discuss and to compare between data sets. If both parameters are needed, as in the case of studying the dynamics of epicotyl emergence of pedunculate oak, both parameterisations can be used. The important advantage of using a model adequate to the needs, that is, containing useful parameters, is the calculation of approximated standard errors and confidence intervals for the estimated parameters directly from the model (Zwietering et al. 1990). If the purpose of the study is to compare the growth curves between data sets (e.g. populations originated from environments of various climatic conditions), the computed confidence intervals will allow to check the significance of differences between them (Tjørve and Tjørve 2017a).

It is worth, or even necessary, to fit more than one growth model for the analysed data set (Tjørve and Tjørve 2017b). In the U-Richards family, each parameter (similar to that in Models 2-3, 5-6 and 8-9) has the same meaning in every model and can, therefore, be compared directly across all models. Having results for several models, we can choose the best model by considering not only criteria of model selection, for example, $\mathrm{AIC}_{\mathrm{c}}$ or $\mathrm{RV}$, but also, importantly, the linear behaviour of parameters. Nonlinear regression models differ greatly amongst themselves in the extent to which their estimators are close to or far from linearity. The growth model with the parameters of important biological meaning would be of little practical use if its estimators were grossly biased (Ratkowsky 1993). Therefore, choosing the best model should be a compromise between the criteria of model selection and the measures of the quality of the model parameter estimators.

\section{Conclusions}

Answers to the questions posed in the Introduction section are very important from the point of view of ecophysiological research of seeds. The applied growth models allow the taking into account of very important parameters, apart from the standard ones such as $T_{50}$ and cumulative germination capacity $A$, and also lag time $T_{\lambda}$ and the maximum absolute growth rate $\mu_{i}$, indicating the dynamics of the germination process. Values of these parameters vary depending on the growth conditions and can serve as a fitness indicator.

All models analysed showed a close-to-linear behaviour of model parameters, but in the present case (epicotyl emergence of oak), the best-fitting model was the Richards model. On the basis of the presented research, we believe that the considered growth models from the Richards family not only are a useful tool for studying the dynamics of the epicotyl emergence of oak but also can be successfully used to study the germination dynamics of trees.

\section{References}

Akaike, H. 1974. A new look at the statistical model identification. IEEE transactions on automatic control, AC-19, 716-723. DOI: 10.1109/TAC.1974.1100705

Ashton, M.S., Larson, B.C. 1996. Germination and seedling growth of Quercus (section Erythrobalanus) across openings in a mixed-deciduous forest of southern New England, USA. Forest Ecology and Management, 80, 81-94. 
Bates, D.M., Watts, D.G. 1980. Relative curvature measures of nonlinearity (with discussion). Journal of the Royal Statistical Society, Series B, 42, $1-25$.

Box, M.J. 1971. Bias in nonlinear estimation (with discussion). Journal of the Royal Statistical Society, Series B, 33, 171-201.

Burnham, K.P., Anderson D.R. 2002. Model selection and multimodel inference: a practical informationtheoretical approach. $2 \mathrm{~d}$ ed. Springer-Verlag, New York.

Dennis, J.E. Jr., Schnabel R.B. 1983. Numerical methods for unconstrained optimization and nonlinear equations. Prentice-Hall, Englewood Cliffs.

Fenner, M., Thompson, K. 2005. The ecology of seeds. Cambridge University Press, Cambridge.

García-De La Cruz, Y., López-Barrera, F., RamosPrado, J.M. 2016. Germination and seedling emergence of four endangered oak species. Madera $y$ Bosques, 22 (2), 77-87. https://www.redalyc.org/ articulo.oa? $\mathrm{id}=61749306006$

Gompertz, B. 1825. On the nature of the function expressive of the law of human mortality, and on a new mode of determining the value of life contingencies. Philosophical Transactions of the Royal Society, 182, 513-585.

Haines, L.M., O’Brien, T.E., Clarke, G.P.Y. 2004. Kurtosis and curvature measures for nonlinear regression models. Statisitica Sinica, 14, 547-570.

Hawkins, T.S. 2018. Regulating acorn germination and seedling emergence in Quercus pagoda (Raf.) as it relates to natural and artificial regeneration. New Forests. DOI: 10.1007/s11056-018-9667-z

Hougaard, P. 1982. Parameterizations of nonlinear models. Journal of the Royal Statistical Society, Series B, 44, 244-252.

Hougaard, P. 1985. The appropriateness of the asymptotic distribution in a nonlinear regression model in relation to curvature. Journal of the Royal Statistical Society, Series B, 47, 103-114.

Kramer, P.J., Kozlowski, T.T. 1979. Physiology of woody plants. Academic Press, New York.

Makeham, W.M. 1873. On the integral of Gompertz's function for expressing the values of sums depending upon the contingency of life. Journal of the Institute of Actuaries and Assurance Magazine, 17 (5), 305-327. DOI: 10.1017/S2046167400044268
Marquardt, D.W. 1963. An algorithm for least squares estimation of nonlinear parameters. Journal of the Society of Industrial Applied Mathematics, 2, 431-441. DOI: 10.1137/0111030

Ratkowsky, D.A. 1983. Nonlinear regression modelling. Marcel Dekker, New York.

Ratkowsky, D.A. 1990. Handbook of nonlinear regression models. Marcel Dekker, New York.

Ratkowsky, D.A. 1993. Principles of nonlinear regression modelling. Journal of Industrial Microbiology and Biotechnology, 12 (3), 195-199. DOI: 10.1007/ BF01584190

Ratkowsky, D.A., Reddy, G.V.P. 2017. Empirical model with excellent statistical properties for describing temperature-dependent developmental rates of insects and mites. Annals of the Entomological Society of America, 110, 302-309. DOI: 10.1093/aesa/saw098

Richards, F.J. 1959. A flexible growth function for empirical use. Journal of Experimental Botany, 10, 290-300. DOI: 10.1093/jxb/10.2.290

SAS Institute, 2017. Inc. SAS/STAT 14.3 User's Guide; SAS Institute Inc.: Cary, NC, USA.

Seber, G.A.F., Wild, C.J. 1989. Nonlinear regression. Wiley \& Sons Publication, New York. ISBN 0-47147135-6

Struve, D.K. 1998. Seed conditioning of red oak: a recalcitrant North American seed. Scientia Agricola, 55 special issue Piracicaba, 67-73. DOI: 10.1590/ S0103-90161998000500012

Tjørve, E., Tjørve, K.M.C. 2010. A unified approach to the Richards-model family for use in growth analyses: Why we need only two model forms. Journal of Theoretical Biology, 267, 417-425. DOI: 10.1016/j. jtbi.2010.09.008 PMID: 20831877

Tjørve, K.M.C., Tjørve, E. 2017a. The use of Gompertz models in growth analyses, and new Gompertzmodel approach: An addition to the Unified-Richards family. PLoS ONE, 12 (6), e0178691. DOI: 10.1371/journal.pone.0178691

Tjørve, K.M.C., Tjørve, E. 2017b. A proposed family of unified models for sigmoidal growth. Ecological Modelling, 359, 117- 127. DOI: 10.1016/j.ecolmodel.2017.05.008

Vrána, J., Remeš, V., Matysioková, B., Tjørve, K.M., Tjørve, E. 2018. Choosing the right sigmoid growth function using the unified-models approach. Ibis. DOI: $10.1111 /$ ibi.12592 
Verhulst, P.F. 1838. A note on population growth (in French). Correspondence Mathematiques et Physiques, 10, 113-121.

Verhulst, P.F. 1845. Mathematical researches into the law of population growth increase (in French). Nou- veaux Mémoires de l'Académie Royale des Sciences et Belles-Lettres de Bruxelles, 18, 1-42.

Zwietering, M.H., Jongenburger, I., Rombouts, F.M. 1990. Modeling of the bacterial growth curve. Applied and Environmental Microbiology, 56, 1875-1881. DOI: 0099-2240/90/061875-07\$02.00/0 